\title{
BMJ Open Accuracy of CT texture analysis for differentiating low-grade and high- grade renal cell carcinoma: systematic review and meta-analysis
}

Wei Yu, Gao Liang, Lichuan Zeng, Yang Yang, Yinghua Wu

To cite: Yu W, Liang G, Zeng L, et al. Accuracy of CT texture analysis for differentiating lowgrade and high-grade renal cell carcinoma: systematic review and meta-analysis. BMJ Open 2021;11:e051470. doi:10.1136/ bmjopen-2021-051470

- Prepublication history and additional supplemental material for this paper are available online. To view these files, please visit the journal online (http://dx.doi.org/10.1136/ bmjopen-2021-051470).

Received 29 March 2021 Accepted 30 November 2021

Check for updates

(c) Author(s) (or their employer(s)) 2021. Re-use permitted under CC BY-NC. No commercial re-use. See rights and permissions. Published by BMJ.

Department of Radiology, Hospital of Chengdu University of Traditional Chinese Medicine, Chengdu, Sichuan, China

Correspondence to

Dr Yinghua Wu;

wuyinghua0319@163.com

\section{ABSTRACT}

Objectives This study aimed to assess the accuracy of CT texture analysis (CTTA) for differentiating low-grade and high-grade renal cell carcinoma (RCC).

Design Systematic review and meta-analysis.

Data sources PubMed, Cochrane Library, Embase, Web of Science, OVID Medline, Science Direct and Springer were searched to identify the included studies.

Eligibility criteria for including studies Clinical studies that report about the accuracy of CTTA in differentiating low-grade and high-grade RCC.

Methods Multiple databases were searched to identify studies from their inception to 20 0ctober 2021. Two radiologists independently extracted data from the primary studies. The pooled sensitivity, specificity, positive likelihood ratio (PLR), negative likelihood ratio (NLR) and diagnostic OR (DOR) were calculated to assess CTTA performance. The summary receiver operating characteristic (SROC) curve was plotted, and the area under the curve (AUC) was calculated to evaluate the accuracy of CTTA in grading RCC.

Results This meta-analysis included 11 studies, with 1603 lesions observed in 1601 patients. Values of the pooled sensitivity, specificity, PLR, NLR, DOR were 0.79 (95\% Cl 0.73 to 0.84$), 0.84$ (95\% Cl 0.81 to 0.87 ), 5.1 (95\% Cl 4.0 to 6.4), 0.24 (95\% Cl 0.19 to 0.32 ) and 21 (95\% Cl 13 to 33), respectively. The SROC curve showed that the AUC was $0.88(95 \% \mathrm{Cl} 0.84$ to 0.90$)$. Deeks' test found no significant publication bias among the studies $(p=0.42)$.

Conclusions The findings of this meta-analysis suggest that CTTA has a high accuracy in differentiating low-grade and high-grade RCC. A standardised methodology and large sample-based study are necessary to certain the diagnostic accuracy of CTTA in RCC grading for clinical decision making.

\section{INTRODUCTION}

Renal cell carcinoma (RCC) is one of the most common malignancies of the urinary system. However, among the main determinants of RCC prognosis, nuclear grading of carcinoma is widely recognised as an important independent factor. ${ }^{1}$ According to the Fuhrman grading system (FGS), RCC is divided into grades I-IV. ${ }^{2}$ Previous studies have shown
Strengths and limitations of this study

- All literature included in this meta-analysis was searched comprehensively from multiple databases.

- Small number of studies were included according to the inclusion and exclusion criteria.

- Standardised methodology and large sample-based studies are necessary to determine the diagnostic accuracy of CT texture analysis in renal cell carcinoma grading.

that the FGS can be considered to be an independent factor for predicting the prognosis of patients with RCC. ${ }^{3}$ In addition, the simplified two-tier FGS has the same accuracy as the four-tier FGS in predicting the prognosis of RCC, with grades I-II being considered low-grade and grades III-IV being considered high-grade. ${ }^{4}$ This simplified grading system reduces inter-observer variability. Low-grade RCC has a high 5-year survival rate, while high-grade RCC has a high metastatic rate and low survival rate. ${ }^{5}$ However, recent studies have revealed that loopholes in this grading system result in poor reproducibility of tumour grading. ${ }^{78}$ The International Society of Urologic Pathology (ISUP) standard proposed a new grading system for RCC at the 2012 ISUP conference, namely the $\mathrm{WHO} /$ ISUP grading system, which was recommended by the WHO in 2016. This grading system can accurately distinguish grades, and it has been shown to be valuable for predicting biological behaviour for clear cell and papillary RCC. ${ }^{9}$ The FGS is based on the simultaneous assessment of nuclear size, nuclear shape and nucleolar prominence. The grading is based on the highest-grade area, even if it is focal. Thus, minute foci of higher-grade RCC, as well as carcinoma adjacent to the foci of necrosis, should be taken into account for grading purposes. It is likely that these problems will result in limited 


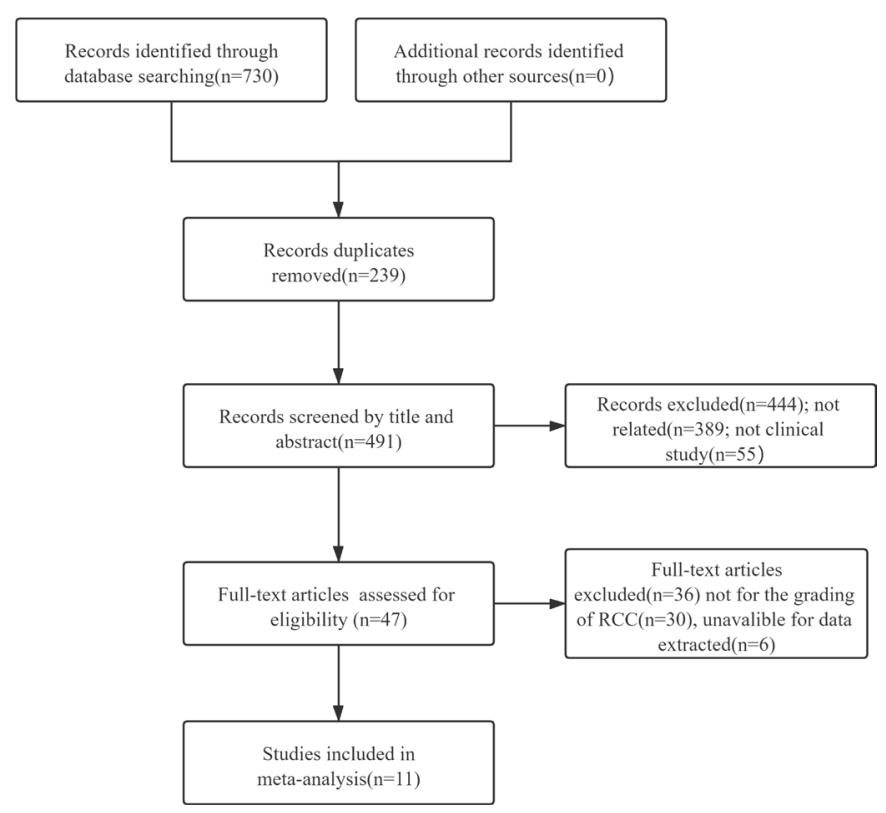

Figure 1 Included studies selection process for this metaanalysis.

interobserver reproducibility. The WHO/ISUP grading system of RCC is based on nucleolar prominence or eosinophilia for grades I-III, while grade IV requires nuclear anaplasia (including tumour giant cells, sarcomatoid differentiation and/or rhabdoid morphology). Thus, the FGS and WHO/ISUP grading systems require histopathological assessment, which is an invasive method. Therefore, it is particularly important to explore a non-invasive method that can accurately assess the nuclear grading of RCC.

Currently, various imaging modalities, such as CT, MRI and positron emission tomography, provide effective methods for the early diagnosis and assessment of RCC prognosis. However, these conventional imaging methods are not specific for predicting the pathological grading of RCC before surgery. With the development of medical imaging technology, texture analysis (TA) has been used for the diagnosis and assessment of RCC grading. TA is a technique for automatically extracting quantitative features from medical images. It can extract hundreds of texture features from every image; thus, it obtains more detailed quantitative information about carcinomas than that of conventional imaging methods. ${ }^{10}$ TA can identify smaller lesions that are macroscopically invisible. Recent studies have shown that TA is generally used for diagnosis, subtype classification, and grading of RCC, which indicates that TA can provide useful information for grading, staging, and predicting prognosis of tumours. Numerous imaging patterns associated with TA, including conventional and contrast-enhanced CT, have been employed to noninvasively and accurately classify RCC grading and to evaluate the heterogeneity of RCC. However, there is no unified conclusion regarding the accuracy of CTTA for differentiating between low-grade and high-grade RCCs. The current meta-analysis aimed to comprehensively and systematically assess the accuracy of CTTA in differentiating low-grade and high-grade RCCs.

\section{MATERIALS AND METHODS}

Patient and public involvement

Patient and the public were not involved in this study.

\section{Searching strategies}

A literature search was independently performed by two radiologists. The databases were searched from their inception to 20 October 2021 including PubMed, Cochrane Library, Embase, Web of Science, OVID Medline, ScienceDirect, and Springer. The search terms were "renal cell carcinoma", "renal cancer", "nephroid carcinoma", "texture analysis", "radiomics", "computed tomography", "CT" and so on. The titles and abstracts were searched for their relevance. The search strategy is presented in detail in online supplemental file 1. Studies were included according to inclusion and exclusion criteria.

\section{Inclusion and exclusion criteria}

Studies were selected according to the following criteria: (1) Clinical studies of CTTA for evaluating the accuracy of differentiating low-grade and high-grade RCC, including diagnostic case-control studies, (2) Data were available and could be extracted for calculating the true positive (TP), false positive (FP), true negative (TN) and false negative (FN) values, (3) Histopathological results were used as the gold standard and (4) English literature. The studies were excluded according to the following criteria: (1) Case reports, reviews, abstracts, meta-analyses or animal studies, (2) The data could not be extracted sufficiently or used to calculate estimates in the study and (3) The grade of RCC was assessed only by medical imaging without pathological confirmation.

\section{Quality assessment of included studies}

The quality of each study was evaluated according to the Quality Assessment of Diagnostic Accuracy Studies (QUADAS-2), ${ }^{11}$ which is recommended by the Cochrane collaboration web.

\section{Data extraction}

Some studies were deemed irrelevant after reading the titles and abstracts and were excluded. The included studies were selected after reading the full texts based on the inclusion and exclusion criteria. Information extracted from the primary study was as follows: the first author, year of publication, country and language, sample size, research type, model used, gold standard, age of patients, TP, FP, FN, TN, CT slice thickness, contrast, speed of injection and segmentation software. Low-grade RCC (grade I-II) was considered positive, while highgrade RCC (grades II-IV) was considered negative.

\section{Meta-analysis}

Meta-analysis was conducted by Review Manager V.5.3, Meta-DiSc V.1.4 (Meta disc, Unit of Clinical Biostatistics 


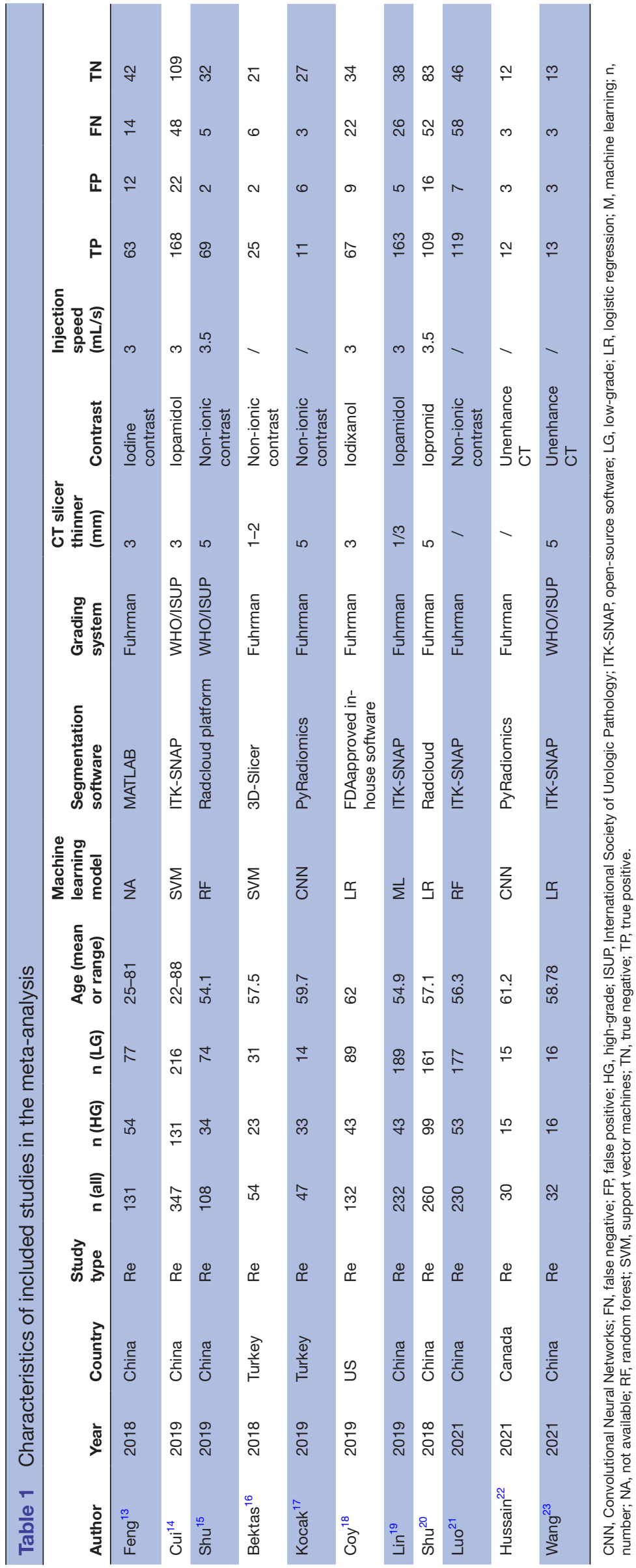


Table 2 Information of six studies with unavailable data

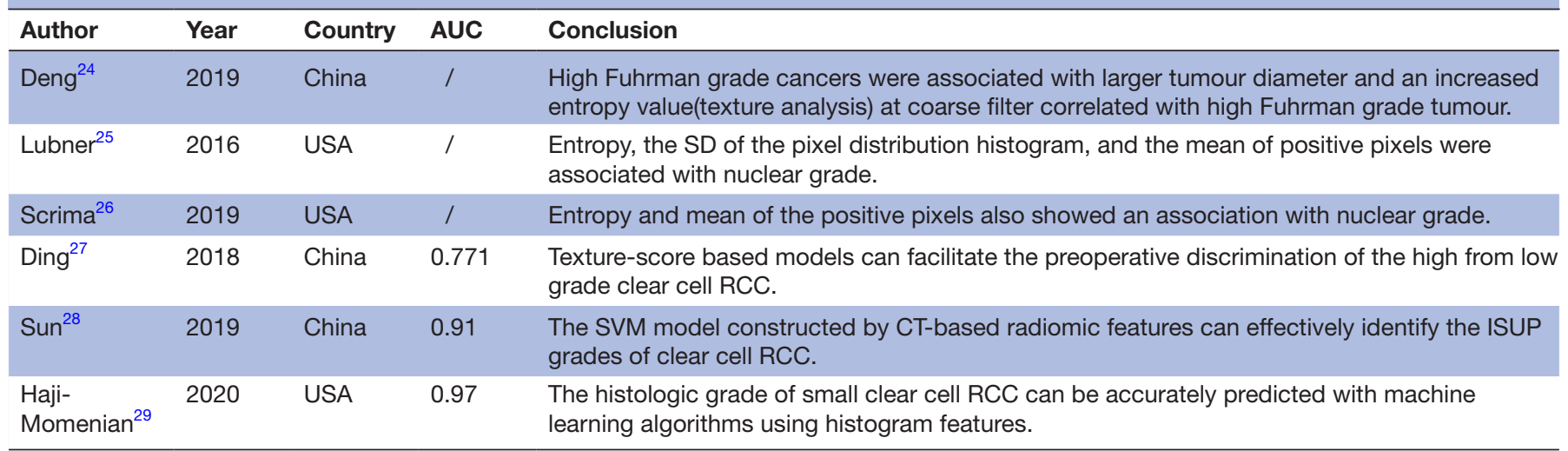

AUC, area under the curve; ISUP, International Society of Urologic Pathology; RCC, renal cell carcinoma; SVM, support vector machines.

of Ramón y Cajal Hospital, Madrid, Spain) and Stata V.15.1. Based on our opinion of the heterogeneity in the extracted data, we adopted a bivariate random effects model to calculate the pooled estimates in advance. The Cochran-Q method and inconsistency index $\left(\mathrm{I}^{2}\right.$ were used to investigate heterogeneity among the studies. If $\mathrm{I}^{2}>50 \%, \mathrm{p}<0.05$, the observed heterogeneity was significant. If $\mathrm{I}^{2}<50 \%, \mathrm{p}>0.05$, the observed heterogeneity was not significant. Pooled sensitivity (Sen), specificity (Spec), PLR, NLR and diagnostic OR (DOR) were calculated to assess the diagnostic performance of CTTA. The summary receiver operating characteristic (SROC) curve was plotted, and the area under the curve (AUC) was calculated. Deeks' test was used to evaluate publication bias, and $p>0.05$, indicating that there was no significant bias. ${ }^{12}$

\section{RESULTS}

\section{Research and selection of studies}

A total of 730 relevant articles were initially identified, and 239 duplicate articles were excluded. Additionally, 444 articles were removed after reading their titles and abstracts and being deemed irrelevant. Subsequently, after reading the full texts, 30 articles were found to be reviews or not related to the grade of RCC, and 6 articles were unavailable for data extraction. Ultimately, after checking for relevant studies of the reference in each review or meta analysis, 11 articles were included. ${ }^{13-23}$ The literature search process is shown in figure 1 . There were six studies in which detailed data were unavailable. $^{24-29}$

\section{Study characteristics}

The characteristics of the included studies are shown in table 1. All 11 studies were retrospective cohort studies. The total number of patients was 1601 with 1603 lesions observed. From the included studies, the age range or mean age of patients were reported and the histology grading system adopted were used as reference standards. The average age of patients in most studies ranged between 54 and 62 years. Two studies reported only the age range of patients without a mean age. Among the included studies, three adopted the WHO/ISUP grading system and eight applied the FGS. The CT slice thickness was $5 \mathrm{~mm}$ and $3 \mathrm{~mm}$ in four and three studies, respectively, while it was $1-2 \mathrm{~mm}$ and $1 \mathrm{~mm}$ or $3 \mathrm{~mm}$ in one study each. The CT slice thickness was not mentioned in the remaining two studies. The information of the six studies with unavailable data is shown in table 2

\section{Quality assessment and publication bias}

The quality of the included studies was evaluated according to the QUADAS-2 checklist, and the results are shown in figures 2 and 3. It was observed that 'index test' in 'risk of bias' and 'applicability concerns' revealed high shortcomings $(2 / 11)$, which may suggest bias regarding inclusion. Overall, the quality of included studies was satisfactory. Deeks' funnel plot asymmetry test was used to assess the

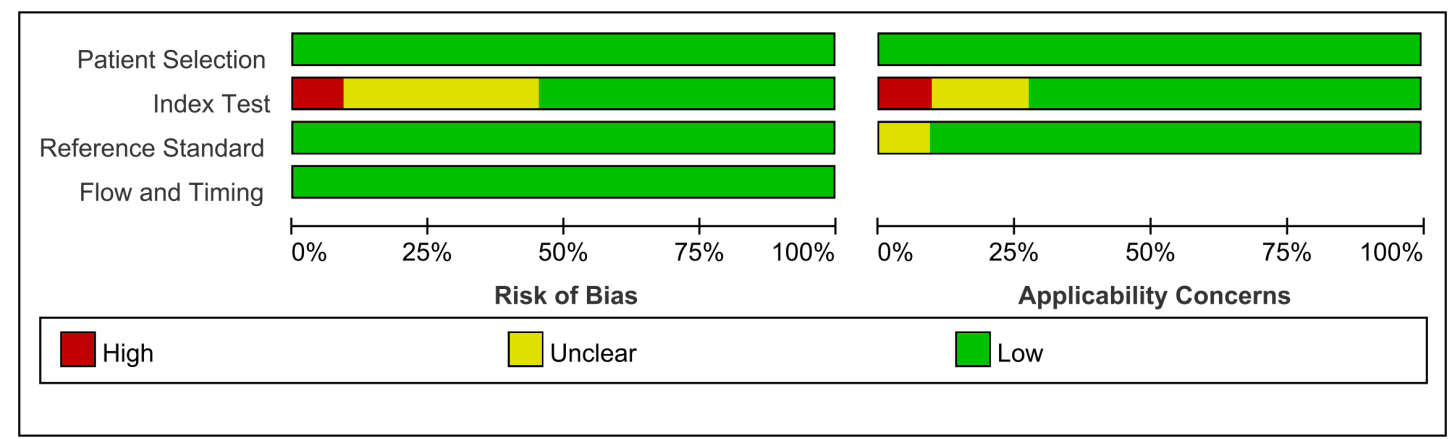

Figure 2 Charts show risk of bias according to QUADAS-2. QUADAS-2, Quality Assessment of Diagnostic Accuracy Studies. 


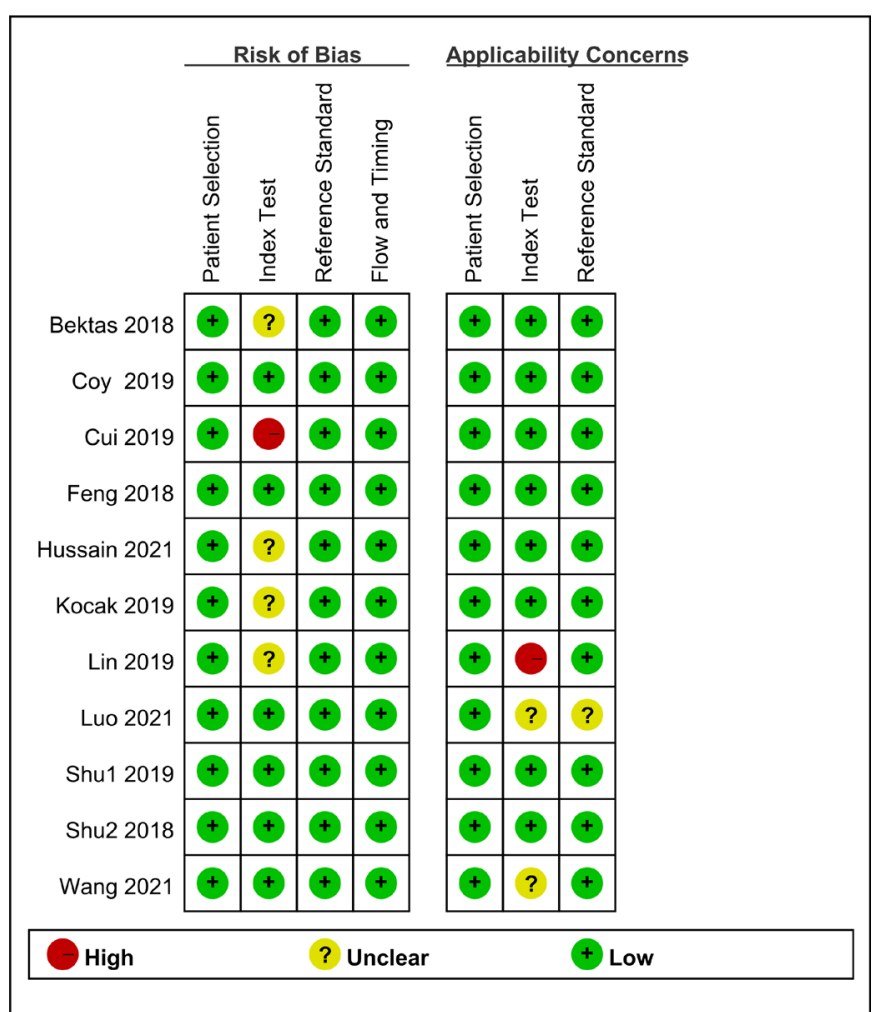

Figure 3 The other plot of charts show risk of bias according to QUADAS-2. QUADAS-2, Quality Assessment of Diagnostic Accuracy Studies.

potential publication bias. The results shown in figure 4 indicate no significant bias $(\mathrm{p}=0.42)$.

\section{Pooled results}

The results of the meta-analysis are presented in figures 5 and 6. Pooled sensitivity and specificity were $0.79(95 \%$ CI 0.73 to 0.84 ) and 0.84 (95\% CI 0.81 to 0.87$)$, respectively (figure 5). Values of PLR, NLR, and DOR were 5.1 (95\% CI 4.0 to 6.4 ), 0.24 (95\% CI 0.19 to 0.32 ), and 21 (95\% CI 13 to $33)$, respectively. The AUC of SROC was 0.88 (95\% CI 0.84 to 0.90) (figure 6). These findings indicate that CTTA has

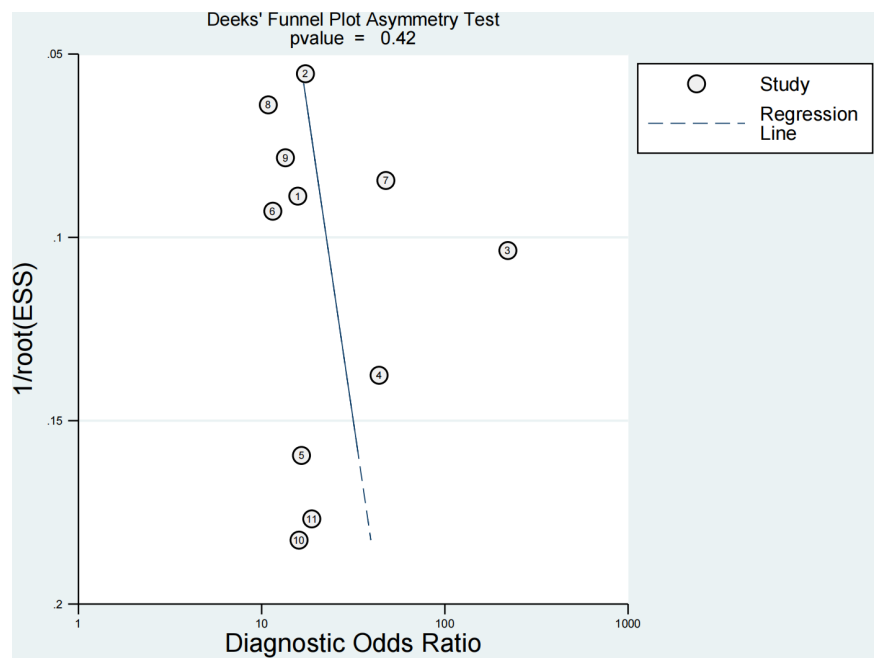

Figure 4 Deeks' funnel plot to test publication bias. a high diagnostic performance in differentiating low-grade and high-grade RCC.

\section{Heterogeneity test}

Spearman correlation analysis was applied to test the threshold effect, which was caused by the use of different diagnostic cut-off values in a single diagnostic test. The Spearman correlation coefficient was $-0.191(\mathrm{p}=0.574)$, indicating that no significant threshold effect was produced. Heterogeneity was tested using Cochran-Q and $\mathrm{I}^{2}$. In figure 5, the $\mathrm{p}$ value of the Cochran-Q test was $0.00(\mathrm{p}<0.05)$, and $\mathrm{I}^{2}$ was $76.39 \%$ in pooled sensitivity. And there was no significant heterogeneity in the pooled specificity $\left(\mathrm{p}>0.05, \mathrm{I}^{2}=0.00 \%\right)$. These results indicated that there was high heterogeneity in pooled sensitivity among the included studies. Thereafter, we used a bivariate random effect meta-regression to explore the potential association of heterogeneity. The results are presented in online supplemental file 2 .

\section{DISCUSSION}

TA technology was first applied to assess the heterogeneity of tumours, and it was considered to have great potential for the evaluation of renal masses. ${ }^{3031}$ In recent years, TA based on CT has been gradually applied to differentiate RCC grades. ${ }^{27}$ However, studies have demonstrated different diagnostic performances of TA in the diagnosis of RCC. The aim of this meta-analysis was to assess the accuracy of CTTA in differentiating between low-grade and high-grade RCC. The values of pooled sensitivity, specificity, PLR, NLR and AUC were $0.79,0.84,5.1,0.24$ and 0.88 , respectively. The results indicated that CTTA had excellent diagnostic performance in differentiating low-income and high-grade RCC, which could be considered a reliable method for diagnosing the grade of RCG in clinical practice.

The gold standard for the diagnosis of RCG is histopathological biopsy. However, this method is invasive and may be inaccurate because of the samples being collected from different biopsy sites. ${ }^{32}$ The field of CTTA, owing to the ability of this technology to quantitatively extract texture features, ${ }^{24}$ has attracted the attention of researchers. It avoids the subjective influence of image processing and reduces the possibility of errors. Currently, the assessment of RCC based on traditional imaging methods primarily includes the overall outline of RCC, such as size, shape and degree of contrast enhancement. However, these parameters can only define the outline and anatomical sites of tumours and are unable to provide vital information regarding the grade of the carcinomas. ${ }^{33}$ The differences between low-grade and high-grade RCC involve changes in the pixel intensity of the images. CTTA can detect subtle changes in pixel intensity caused by heterogeneity between low-grade and high-grade RCC. In addition, CTTA performs a comprehensive evaluation of lesions. Compared with biopsy, this technique evaluates the mass through an integrated rather than a focal analysis, which avoids the influence of sampling site error. Different grades of RCC require different therapies. Lowgrade RCG patients may undergo partial nephrectomy, 

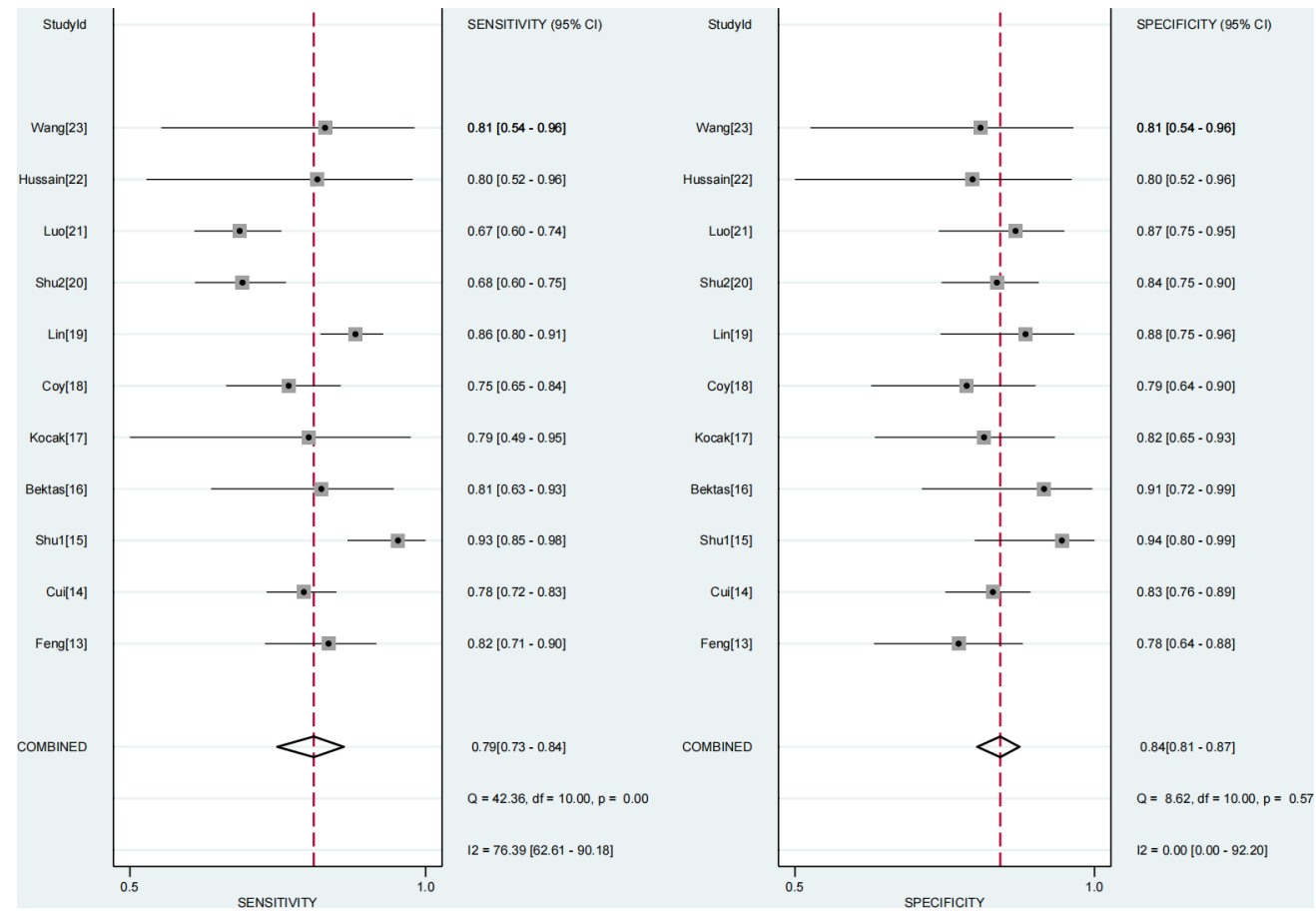

Figure 5 Coupled forest plots of sensitivity and specificity of CTTA for differentiating between low-grade and high-grade RCC. CTTA, CT texture analysis; RCC, renal cell carcinoma.

whereas high-grade RCC patients may require more invasive and extensive surgery. ${ }^{34}$ As an important prognostic factor, it is important to preoperatively differentiate the grade of RCC in clinical practice and provide more valuable guidance for clinicians.

Image preprocessing is an essential step in TA, and the methods used for image preprocessing differed greatly in the included studies. Image segmentation and quantitative

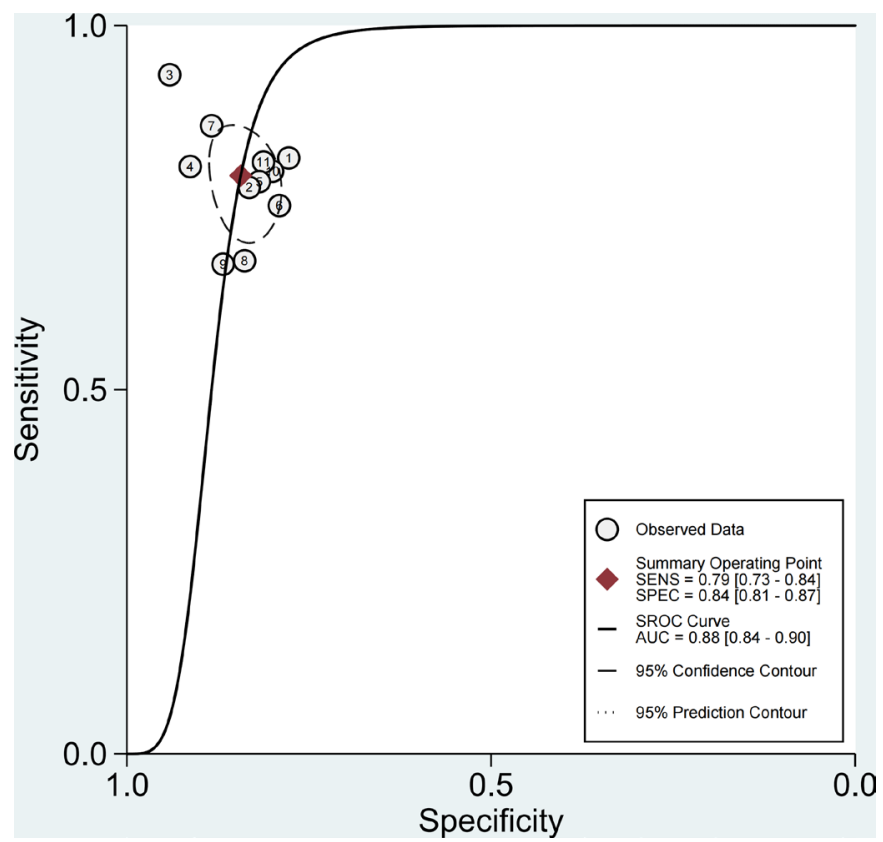

Figure 6 Summary receiver operating characteristics (SROC) curve to differentiate low-grade and high-grade RCC. $A \cup C$, area under the curve; RCC, renal cell carcinoma; SENS, sensitivity; SPEC, specificity. analysis were conducted after the image preprocessing step, ${ }^{35}$ followed by the establishment of a diagnostic predictive model. Lastly, the SROC curve and AUC were calculated to evaluate diagnostic performance. Previous studies have indicated that texture features, such as entropy, SD and mean of the positive pixels, were associated with nuclear grade. An increased entropy value correlated with high Fuhrman grade tumours. ${ }^{24-26}$ The radiomics model with texture features constructed by some scholars indicated a high prediction accuracy in identifying the grading of RCC. ${ }^{27-29}$

There were some limitations in this meta-analysis: all included studies in this meta-analysis were retrospective in design, which has a higher bias risk than prospective studies; there was high heterogeneity between the included studies, which may be due to the age of patients, tools of TA and image preprocessing; some of the included studies were conducted with a small number of samples; and the sample size varied greatly, which may affect the accuracy of the results.

\section{CONCLUSION}

This study suggested that CTTA has high accuracy in differentiating low-grade and high-grade RCC, which could be considered as a non-invasive method to provide crucial information for the grading of RCC. However, a standard methodology and large sample-based study are necessary to certain the diagnostic accuracy of CTTA in RCC grading.

Contributors WY and GL came up with this meta-analysis and completed the work of design. $L Z$ and $Y Y$ implemented this systematic review with the guidence of WY. LZ and YY performed the literature search, data extracion and quality assessment. Statistical analysis was completed by WY and YW. YW is the guarantor 
for this article. WY and GL wrote the first draft, then all authors participated in the modification of the manuscript.

Funding The authors have not declared a specific grant for this research from any funding agency in the public, commercial or not-for-profit sectors.

Competing interests None declared.

Patient consent for publication Not applicable.

Ethics approval This study does not involve human participants.

Provenance and peer review Not commissioned; externally peer reviewed.

Data availability statement All data relevant to the study are included in the article or uploaded as online supplemental information.

Supplemental material This content has been supplied by the author(s). It has not been vetted by BMJ Publishing Group Limited (BMJ) and may not have been peer-reviewed. Any opinions or recommendations discussed are solely those of the author(s) and are not endorsed by BMJ. BMJ disclaims all liability and responsibility arising from any reliance placed on the content. Where the content includes any translated material, BMJ does not warrant the accuracy and reliability of the translations (including but not limited to local regulations, clinical guidelines, terminology, drug names and drug dosages), and is not responsible for any error and/or omissions arising from translation and adaptation or otherwise.

Open access This is an open access article distributed in accordance with the Creative Commons Attribution Non Commercial (CC BY-NC 4.0) license, which permits others to distribute, remix, adapt, build upon this work non-commercially, and license their derivative works on different terms, provided the original work is properly cited, appropriate credit is given, any changes made indicated, and the use is non-commercial. See: http://creativecommons.org/licenses/by-nc/4.0/.

\section{ORCID iD}

Yinghua Wu http://orcid.org/0000-0001-9116-1624

\section{REFERENCES}

1 Mancini V, Battaglia M, Ditonno P, et al. Current insights in renal cell cancer pathology. Urol Oncol 2008;26:225-38.

2 Fuhrman SA, Lasky LC, Limas C. Prognostic significance of morphologic parameters in renal cell carcinoma. Am J Surg Pathol 1982;6:333-6.

3 Novara G, Martignoni G, Artibani W, et al. Grading systems in renal cell carcinoma. J Urol 2007;177:1172-3.

4 Smith ZL, Pietzak EJ, Meise CK, et al. Simplification of the Fuhrman grading system for renal cell carcinoma. Can J Urol 2015;22:8069-73.

5 Becker A, Hickmann D, Hansen J, et al. Critical analysis of a simplified Fuhrman grading scheme for prediction of cancer specific mortality in patients with clear cell renal cell carcinoma--Impact on prognosis. Eur J Surg Oncol 2016;42:419-25.

6 Al-Aynati M, Chen V, Salama S, et al. Interobserver and intraobserver variability using the Fuhrman grading system for renal cell carcinoma. Arch Pathol Lab Med 2003;127:593-6.

7 Delahunt B, Egevad L, Samaratunga $\mathrm{H}$, et al. Gleason and Fuhrman no longer make the grade. Histopathology 2016;68:475-81.

8 Delahunt B, Sika-Paotonu D, Bethwaite PB, et al. Grading of clear cell renal cell carcinoma should be based on nucleolar prominence. Am J Surg Pathol 2011;35:1134-9.

9 Moch $\mathrm{H}$. [The WHO/ISUP grading system for renal carcinoma]. Pathologe 2016;37:355-69.

10 Lambin P, Rios-Velazquez E, Leijenaar R, et al. Radiomics: extracting more information from medical images using advanced feature analysis. Eur J Cancer 2012;48:441-6.

11 Whiting PF, Rutjes AWS, Westwood ME, et al. QUADAS-2: a revised tool for the quality assessment of diagnostic accuracy studies. Ann Intern Med 2011;155:529-36.

12 Deeks JJ, Macaskill P, Irwig L. The performance of tests of publication bias and other sample size effects in systematic reviews of diagnostic test accuracy was assessed. $J$ Clin Epidemiol 2005;58:882-93.

13 Feng Z, Shen Q, Li Y, et al. Ct texture analysis: a potential tool for predicting the Fuhrman grade of clear-cell renal carcinoma. Cancer Imaging 2019;19:6-9.
14 Cui E-M, Lin F, Li Q, et al. Differentiation of renal angiomyolipoma without visible fat from renal cell carcinoma by machine learning based on whole-tumor computed tomography texture features. Acta Radiol 2019;60:1543-52.

15 Shu J, Wen D, Xi Y, et al. Clear cell renal cell carcinoma: machine learning-based computed tomography radiomics analysis for the prediction of WHO/ISUP grade. Eur J Radiol 2019;121:108738.

16 Bektas CT, Kocak B, Yardimci AH, et al. Clear cell renal cell carcinoma: machine Learning-Based quantitative computed tomography texture analysis for prediction of Fuhrman nuclear grade. Eur Radiol 2019;29:1153-63.

17 Kocak B, Ates E, Durmaz ES, et al. Influence of segmentation margin on machine learning-based high-dimensional quantitative CT texture analysis: a reproducibility study on renal clear cell carcinomas. Eur Radiol 2019;29:4765-75.

18 Coy $\mathrm{H}$, Young JR, Pantuck AJ, et al. Association of tumor grade, enhancement on multiphasic CT and microvessel density in patients with clear cell renal cell carcinoma. Abdom Radiol 2020;45:3184-92.

19 Lin F, Cui E-M, Lei Y, et al. CT-Based machine learning model to predict the Fuhrman nuclear grade of clear cell renal cell carcinoma. Abdom Radiol 2019;44:2528-34.

20 Shu J, Tang Y, Cui J, et al. Clear cell renal cell carcinoma: CT-based radiomics features for the prediction of Fuhrman grade. Eur $J$ Radiol 2018;109:8-12

21 Luo S, Wei R, Lu S, et al. Fuhrman nuclear grade prediction of clear cell renal cell carcinoma: influence of volume of interest delineation strategies on machine learning-based dynamic enhanced CT radiomics analysis. Eur Radiol 2021. doi:10.1007/s00330-02108322-w. [Epub ahead of print: 12 Oct 2021].

22 Hussain MA, Hamarneh G, Garbi R. Learnable image histogramsbased deep radiomics for renal cell carcinoma grading and staging. Comput Med Imaging Graph 2021;90:101924.

23 Wang $\mathrm{X}$, Song $\mathrm{G}$, Jiang $\mathrm{H}$, et al. Can texture analysis based on single unenhanced CT accurately predict the WHO/ISUP grading of localized clear cell renal cell carcinoma? Abdom Radiol 2021;46:4289-300

24 Deng Y, Soule E, Samuel A, et al. Ct texture analysis in the differentiation of major renal cell carcinoma subtypes and correlation with Fuhrman grade. Eur Radiol 2019;29:6922-9.

25 Lubner MG, Stabo N, Abel EJ, et al. Ct textural analysis of large primary renal cell carcinomas: pretreatment tumor heterogeneity correlates with histologic findings and clinical outcomes. AJR Am J Roentgenol 2016;207:96-105.

26 Scrima AT, Lubner MG, Abel EJ, et al. Texture analysis of small renal cell carcinomas at MDCT for predicting relevant histologic and protein biomarkers. Abdom Radiol 2019;44:1999-2008.

27 Ding J, Xing Z, Jiang Z, et al. CT-based radiomic model predicts high grade of clear cell renal cell carcinoma. Eur J Radiol 2018;103:51-6.

28 Sun X, Liu L, Xu K, et al. Prediction of ISUP grading of clear cell renal cell carcinoma using support vector machine model based on CT images. Medicine 2019;98:e15022.

29 Haji-Momenian S, Lin Z, Patel B, et al. Texture analysis and machine learning algorithms accurately predict histologic grade in small $(<4 \mathrm{~cm})$ clear cell renal cell carcinomas: a pilot study. Abdom Radiol 2020;45:789-98.

30 Ganeshan B, Skogen K, Pressney I, et al. Tumour heterogeneity in oesophageal cancer assessed by CT texture analysis: preliminary evidence of an association with tumour metabolism, stage, and survival. Clin Radiol 2012;67:157-64.

31 Raman SP, Chen Y, Schroeder JL, et al. Ct texture analysis of renal masses: pilot study using random forest classification for prediction of pathology. Acad Radiol 2014;21:1587-96.

32 Marconi L, Dabestani S, Lam TB, et al. Systematic review and metaanalysis of diagnostic accuracy of percutaneous renal tumour biopsy. Eur Urol 2016;69:660-73.

33 Kierans AS, Rusinek H, Lee A, et al. Textural differences in apparent diffusion coefficient between low- and high-stage clear cell renal cell carcinoma. AJR Am J Roentgenol 2014;203:W637-44.

34 Sundeep A BHN, Mohammadhadi B, et al. Eligibility and radiologic assessment for adjuvant clinical trials in kidney cancer. JAMA oncology 2019:132-6.

35 Lewis MA, Ganeshan B, Barnes A, et al. Filtration-histogram based magnetic resonance texture analysis (MRTA) for glioma IDH and 1p19q genotyping. Eur J Radiol 2019;113:116-23. 